\title{
Thermodynamics of Gauss-Bonnet black holes revisited
}

\author{
Yun Soo Myung ${ }^{1, a}$, Yong-Wan Kim ${ }^{1, b}$, and Young-Jai Park ${ }^{2, c}$ \\ ${ }^{1}$ Institute of Basic Science and School of Computer Aided Science, \\ Inje University, Gimhae 621-749, Korea \\ ${ }^{2}$ Department of Physics, Sogang University, Seoul 121-742, Korea
}

\begin{abstract}
We investigate the Gauss-Bonnet black hole in five dimensional anti-de Sitter spacetimes (GBAdS). We analyze all thermodynamic quantities of the GBAdS, which is characterized by the Gauss-Bonnet coupling $c$ and mass $M$, comparing with those of the Born-Infeld-AdS (BIAdS), Reissner-Norström-AdS black holes (RNAdS), SchwarzschildAdS (SAdS), and BTZ black holes. For $c<0$ we cannot obtain the black hole with positively definite thermodynamic quantities of mass, temperature, and entropy because the entropy does not satisfy the area-law. On the other hand, for $c>0$, we find the BIAdS-like black hole, showing that the coupling $c$ plays the role of pseudo-charge. Importantly, we could not obtain the SAdS in the limits of $c \rightarrow 0$, which means that the GBAdS is basically different from the SAdS. In addition, we clarify the connections between thermodynamic and dynamical stability. Finally, we also conjecture that if a black hole is big and thus globally stable, its quasinormal modes may take analytic expressions.
\end{abstract}

PACS numbers: 04.70.Dy, 04.50.Gh, 04.70.-s.

Keywords: Gauss-Bonnet black holes; Thermodynamics; Dynamical stability.

${ }^{a}$ ysmyung@inje.ac.kr

bywkim65@gmail.com

cyjpark@sogang.ac.kr 


\section{Introduction}

Black holes are very important objects in classical and quantum gravity. One of the most important and profound properties in black hole physics is its thermodynamics. Since the black hole thermodynamics has a deep connection with quantum mechanics of gravity, we have a natural question whether the properties related with the black hole thermodynamics are modified, if we add higher dimensional corrections to Einstein-Hilbert action, which are expected to appear in an effective theory of quantum gravity, for example, string theory.

The Gauss-Bonnet (GB) term is the lowest dimensional term among higher dimensional correction ones. The spherically symmetric black hole solutions are known [1], and the thermodynamics is also calculated [2] in Einstein-Gauss-Bonnet (EGB) theory. In particular, in the nontrivial lowest five dimensions, the most general theory of gravity leading to second order field equations for the metric is the EGB theory with the coupling constant c. The GB term with $c=1 / 2 \pi \alpha^{\prime}>0$ appears as the first curvature stringy correction to general relativity [3, 4], when assuming that the tension $\alpha^{\prime}$ of a string is large as compared to the energy scale of other variables. Recently, the study of black holes with higher derivative curvature in anti-de Sitter (AdS) spaces has been considered by many authors. Static AdS black hole solutions in EGB gravity denote GBAdS in this work, presenting a number of interesting features (see e.g. [5], [6], [7] and the references therein).

Since the pioneering work of Hawking-Page phase transition (HP2) between thermal AdS and SAdS in four dimensions [8], the research of the black hole thermodynamics has recently improved. Moreover, the HP2 in five dimensions was discussed for string theories [9]. In the HP2, one generally starts with thermal radiation in AdS space appearing a small black hole with negative heat capacity $\left(\mathrm{SBH}_{-}\right)$. Then, since the heat capacity changes from negative infinity to positive infinity at the minimum temperature, the large black hole with positive heat capacity $\left(\mathrm{LBH}_{+}\right)$finally comes out as a stable object. Evidently, there is a change of the dominance at the critical temperature: from thermal radiation to black hole [8]. On the other hand, it was suggested that there exists a different phase transition (HP1) between the small black hole with positive heat capacity $\left(\mathrm{SBH}_{+}\right)$and $\mathrm{LBH}_{+}$in the RNAdS for fixed charge $Q<Q_{c}[10,11,12]$ and GBAdS [6, 13].

Recently, we have obtained all thermodynamic quantities of the 3D EinsteinBorn-Infeld black holes, which are nonlinear generalization of BTZ black 
holes [14]. Furthermore, we have proposed that the 4D Born-Infeld-anti-de Sitter black holes (BIAdS) with the coupling constant $b$ have the main feature of RNAdS with charge $Q$ : two horizons and a degenerate horizon because the BIAdS is a nonlinear generalization of the RNAdS and disconnects to the SAdS [15]. These studies may enhance the level of understanding the GBAdS.

On the other hand, some issues of 5D Gauss-Bonnet black holes remain unclarified. These include thermodynamic stability, dynamical stability, and the existence of quasinormal (QN) modes [16, 17, 18, 19, 20, 21, 22, 23, 24]. Importantly, their connection known as the correlated stability conjecture was not clearly understood until now because the sign of coupling $c$ was not taken seriously for these analysis. String theories always predict positive coupling of $c>0$, while the negative coupling of $c<0$ is also available to study their black holes. At the first sight, the former may give rise to a black hole like the RNAdS with the charge $c$, while the latter may provide the SAdS with negative mass $c$. However, this interpretation of $c$ is not correct. For $c>0$ case, the $b Q=0.5$ BIAdS will be used to study the $0<c<l^{2} / 36$ GBAdS because their thermodynamic properties are the nearly same. In this case, $c$ plays the role of a pseudo-charge. The role of charge " $c$ " becomes clear when considering the GBRNAdS black hole. For $c<0$, the role of negative mass " $c$ " becomes clear when introducing the $k=-1$ topological GBAdS (TGBAdS).

In this paper, we address these issues for the GBAdS in five dimensions. We revisit all thermodynamic quantities of the GBAdS, which is characterized by the GB coupling $c$ and mass $M$, comparing with those of the BIAdS, RNAdS, SAdS, and BTZ black holes. The similar and related works on the thermodynamics of the GBAdS were carried out in Ref. [13, 25]. We point out that the thermodynamic properties of GBAdS are closely related to their dynamical stabilities and the expressions of QN frequencies.

The organization of this work is as follows. In Sec. 2, we analyze the possible GBAdS black hole solutions depending on the GB coupling $c$. In Sec. 3, we revisit all thermodynamic properties of the GBAdS by comparing those of the BIAdS, RNAdS, SAdS, and BTZ black holes. We also analyze the thermodynamic stability comparing with the approaches of the dynamic stability and quasi-normal modes in the GBAdS black holes in Sec. 4 and Sec. 5, respectively. Finally, we summarize and discuss our results in Sec. 6. In Appendix A, we comment on thermodynamic properties of the $k=$ -1 TGBAdS. In Appendix B, we mention thermodynamic properties of the GBRNAdS black hole. 


\section{Structure of TGBAdS black holes}

Now, let us consider a 5D gravitational action in the presence of a negative cosmological constant $\Lambda=-6 / l^{2}$ and GB term as

$$
I=\frac{1}{16 \pi G_{5}} \int d^{5} x \sqrt{-g}\left[R-2 \Lambda+\frac{c}{2} L_{G B}\right]
$$

where

$$
L_{G B}=R^{2}-4 R_{\mu \nu} R^{\mu \nu}+R_{\mu \nu \rho \sigma} R^{\mu \nu \rho \sigma} .
$$

Here $G_{5}$ is the Newton constant and $c$ is a GB coupling constant having mass dimension -2 . In this work, we consider both the cases with $c>0$ and $c<0$ comparing with the $c=0$ case of SAdS. This action possesses black hole solutions, which we call GBAdS [1, 6, 7, 26, 27, 28, 29].

Varying the action (1) with respect to the gravitational field $g_{\mu \nu}$, the field equation is obtained as follows

$$
R_{\mu \nu}-\frac{1}{2} R g_{\mu \nu}+\Lambda g_{\mu \nu}+\frac{c}{2} H_{\mu \nu}=0
$$

where

$$
H_{\mu \nu}=2\left(R_{\mu \sigma \kappa \tau} R_{\nu}^{\sigma \kappa \tau}-2 R_{\mu \rho \nu \sigma} R^{\rho \sigma}-2 R_{\mu \sigma} R_{\nu}^{\sigma}+R R_{\mu \nu}\right)-\frac{1}{2} L_{G B} g_{\mu \nu} .
$$

By solving the Einstein equation (3), the solution of the TGBAdS black hole is given by [1]

$$
d s^{2}=-f(r) d t^{2}+\frac{d r^{2}}{f(r)}+r^{2} d \Omega_{3}^{2}
$$

The metric function with two branches $\epsilon= \pm 1$ is given by

$$
f(r)=k+\frac{r^{2}}{2 c}\left[1+\epsilon \sqrt{1+\frac{4 c}{3}\left(\frac{2 \mu}{r^{4}}-\frac{3}{l^{2}}\right)}\right],
$$

where the signature $k$ classifies the horizon geometry depending on $k=-1$ (hyperbolic), 0 (flat), 1 (spherical) [28, 29, 30]. Here $\mu$ is the mass parameter related to the black hole mass $M=\mu \mathcal{A}_{3} / 8 \pi G_{5}$ where $\mathcal{A}_{3}=2 \pi^{2}$ is the area of unit three sphere. The GB black hole solution with $k=1$ spherical horizon was first found by Boulware and Deser [1]. Note that although our conventions follow Ref. [25], there exits a clear correspondence to Ref. [13]: $c \rightarrow \tilde{\alpha}, \mu \rightarrow 3 m / 2$.

On the other hand, the metric (5) goes to AdS space asymptotically. In the limit of $r \rightarrow \infty$, the metric function takes the form as

$$
f_{\infty}(r)=\frac{r^{2}}{2 c}\left(1+\epsilon \sqrt{1-\frac{4 c}{l^{2}}}\right) \equiv \frac{r^{2}}{l_{\text {eff }}^{2}}
$$


with the effective $\mathrm{AdS}_{5}$ curvature radius

$$
l_{\text {eff }}^{2}=\frac{l^{2}}{2}\left[1-\epsilon \sqrt{1-\frac{4 c}{l^{2}}}\right] .
$$

We note that this metric is well-defined on the boundary at the infinity if

$$
c \leq l^{2} / 4
$$

Therefore, one has to consider $c$ satisfying the above bound for the $c>0$ case with $\epsilon= \pm 1$, while the $\epsilon=-1$ solution is only possible without any bound for the case of $c<0$.

\section{Thermodynamics of GBAdS black holes}

From now on, we consider the $k=1$ with $\epsilon=-1$, while the $k=-1$ case will be treated in Appendix A. The zeros of $f(r)$ determine the locations of the horizons. In the five dimensions, there is a single horizon at

$$
r_{h}^{2}(\mu, c)=\frac{l^{2}}{2}\left(-1+\sqrt{1+\frac{4(2 \mu-3 c)}{3 l^{2}}}\right) .
$$

We note that in order to have a real solution $r_{h} \geq 0, \mu \geq \frac{3}{2} c$ is required for the case of $c>0$. Otherwise, there is no event horizon for black hole. Now, let us derive the mass parameter as a function of the horizon radius $r_{h}$. From Eq. (10), we have the mass parameter

$$
\mu\left(r_{h}, c\right)=\frac{3}{2}\left(\frac{r_{h}^{4}}{l^{2}}+r_{h}^{2}+c\right)
$$

In order to obtain the structure of the GBAdS, we wish to find the extremal black hole. From the conditions of $f=0$ and $f^{\prime}=0$, we find that the degenerate horizon is located at

$$
r_{e} \equiv r_{h}=0
$$

which is confirmed by noting $r_{h}^{2} \rightarrow 0$ as $\mu \rightarrow 3 c / 2$ in Eq. (10). In this case, the mass parameter is given by

$$
\mu_{e} \equiv \mu(0, c)=\frac{3}{2} c .
$$


(a)

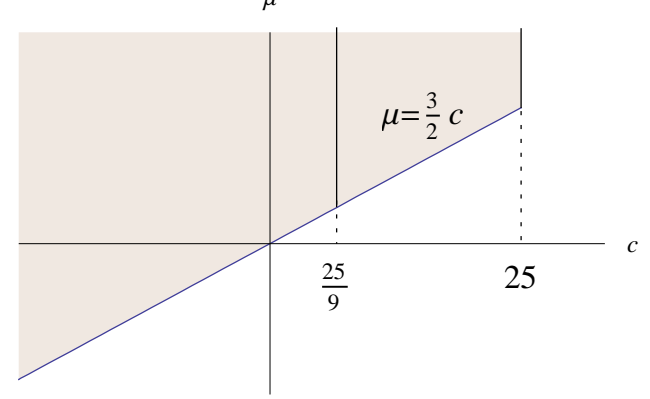

(b)

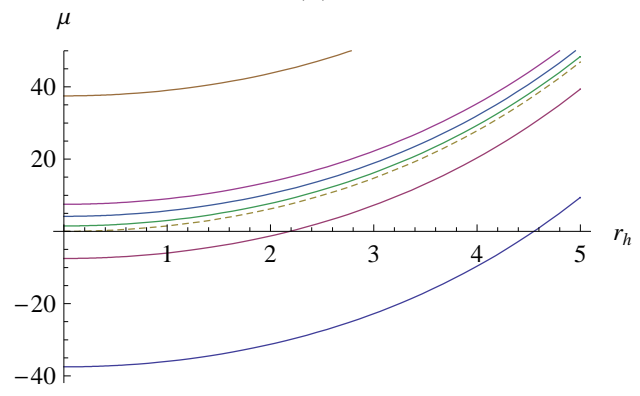

Figure 1: (a) The mass function graph $\mu$ vs $c$ with $l=10 . \quad \mu=3 c / 2$ is the lower bound for $c<0$, while it corresponds to the extremal black hole for $c>0$. The naked singularity regions (NS) are white areas below solid oblique of $\mu=3 c / 2$ and the forbidden region appears for $c>l^{2} / 4$. (b) The mass function graphs $\mu$ vs $r_{h}$ with $l=10$ from top to bottom: $c=25$ (upper bound), 5, 25/9, 1,0(SAdS), $-5,-25$. For the case of $c>0$, we have always positive mass and the extremal black holes at $r_{h}=0$. For $c<0$, we have negative, zero, and positive masses.

Here we point out that $\mu_{e}$ is a mass gab [6] as well as a mass of the extremal GBAdS. Then, the bound of mass is given by

$$
\mu_{e} \leq \mu\left(r_{h}, c\right) \leq \mu\left(r_{h}, \frac{l^{2}}{4}\right) .
$$

On the other hand, for the $c<0$ case, there is no extremal black hole, but there is a bound for mass

$$
\mu \geq-\frac{3}{2}|c|
$$

Here we have negative mass of $\mu<0$ for $r_{h}<l_{0}$ with $l_{0}^{2} \equiv\left(l^{2} / 2\right)[-1+$ $\left.\sqrt{1-4 c / l^{2}}\right]$, zero mass of $\mu=0$ at $r_{h}=l_{0}$, and positive mass of $\mu>0$ for $r_{h}>l_{0}$ as depicted in Fig. 1 .

Now, we are ready to drive the thermodynamic quantities of GBAdS. The Hawking temperature defined by $T_{H}=f^{\prime}\left(r_{h}\right) / 4 \pi$ takes the form

$$
T_{H}\left(r_{h}, c\right)=\frac{r_{h}^{3}}{\pi l^{2}\left(r_{h}^{2}+2 c\right)}\left(1+\frac{l^{2}}{2 r_{h}^{2}}\right) .
$$

Note that from the condition $T_{H}\left(r_{h}, c\right) \geq 0, c$ should satisfy the condition $c>0$ or $r_{h}^{2} \geq-2 c$ for the case of $c<0$. Then, using the Eqs. (11) and (16), the heat capacity defined by $C\left(r_{h}, c\right)=\left(d M / d T_{H}\right)_{c}$ is obtained to be

$$
C=\frac{3 \mathcal{A}_{3}}{4 G_{5}}\left[\frac{r_{h}\left(2 r_{h}^{2}+l^{2}\right)\left(r_{h}^{2}+2 c\right)^{2}}{r_{h}^{2}\left(2 r_{h}^{2}-l^{2}\right)+2 c\left(6 r_{h}^{2}+l^{2}\right)}\right] .
$$



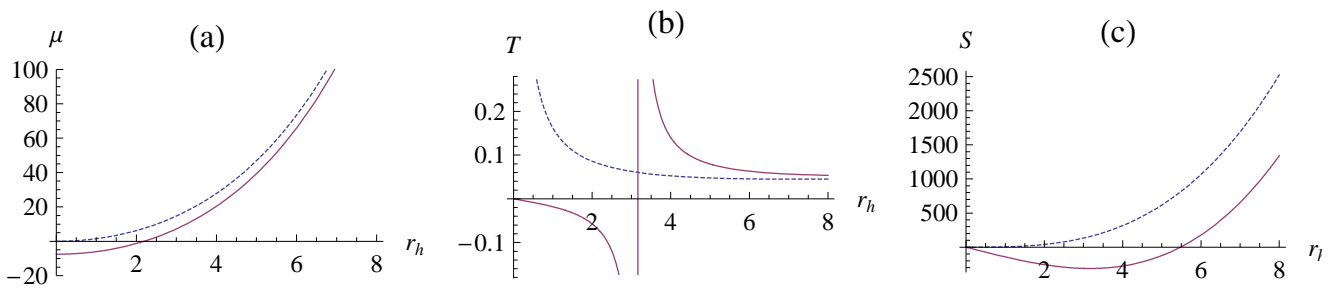

Figure 2: For $c=-5$ GBAdS black hole, improper thermodynamic quantities (the solid curves). (a) $\mu$ vs $r_{h}$ (b) $T$ vs $r_{h}$, and (c) $S$ vs $r_{h}$. All show negative behaviors, which are obstacles to define a proper black hole for $c<0$ thermodynamically. The dotted curves stand for the SAdS, showing good features.

The heat capacity is very important to test the thermodynamic stability of the black hole: if $C>0$, the black hole is thermodynamically stable, while for $C<0$, the corresponding black hole is unstable. If $C>0$, the global stability is guaranteed by the condition of a negative free energy $F<0$. On the other hand, the Bekenstein-Hawking entropy derived from the first-law of thermodynamics takes the form [31]

$$
S_{B H}=\int \frac{d M}{T}=\int_{0}^{r_{h}} \frac{d r_{h}}{T}\left(\frac{d M}{d r_{h}}\right)=\frac{\mathcal{A}_{3} r_{h}^{3}}{4 G_{5}}\left(1+\frac{6 c}{r_{h}^{2}}\right),
$$

which shows obviously that the area-law of the entropy does not hold for the GBAdS. Furthermore, we have always a negative entropy for the $c<0$ case. On the other hand, the on-shell free energy defined by $F\left(r_{h}, c\right)=$ $M-M_{e}-T_{H} S_{B H}$ is given by

$$
F=-\frac{\mathcal{A}_{3}}{16 \pi G_{5}\left(r_{h}^{2}+2 c\right)}\left[\frac{r_{h}^{6}}{l^{2}}-r_{h}^{4}+3 c\left(\frac{6 r_{h}^{4}}{l^{2}}+r_{h}^{2}-2 c\right)\right]-\frac{\mathcal{A}_{3}}{16 \pi G_{5}} c .
$$

Hereafter, we consider two cases of $c<0$ and $c>0$ separately because these provide two distinct branches. Actually, we show that " $c$ " plays the role of negative mass for $c<0$, whereas " $c$ " plays the role of pseudo-charge for $c>0$. However, if one considers $c$ as the pseudo-charge, the extremal mass $M_{e}$ should be included as the ground state in defining the free energy of Eq. (19) in the canonical ensemble [10] similar to the RNAdS case. This picture is important to understand the nature of black holes obtained from the GB term. 
(a)

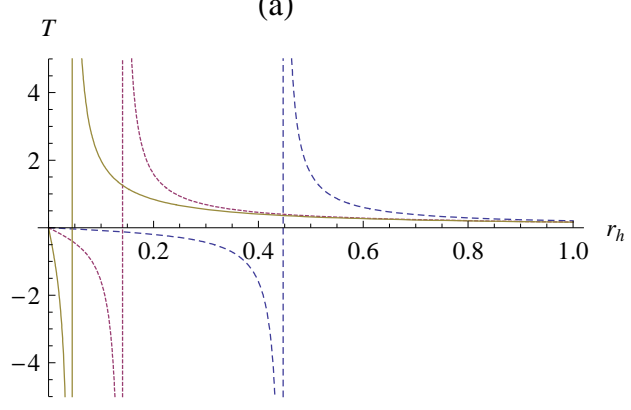

(b)

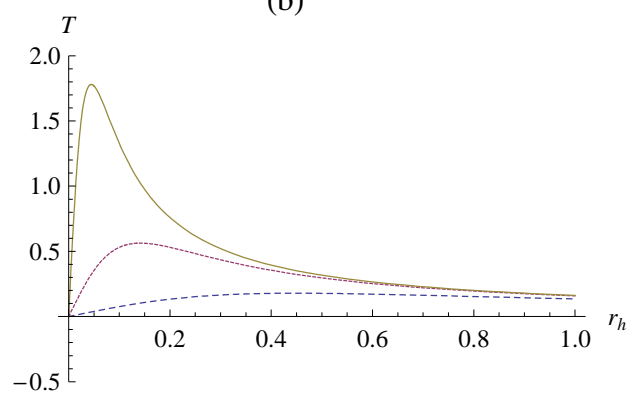

Figure 3: The graphs of temperature vs horizon radius, showing that we cannot obtain the SAdS in the limits of $c \rightarrow 0^{\mp}$. (a) $c=-0.1$ for dashed, $c=-0.01$ for dotted, and $c=-0.001$ for solid curves. (b) $c=0.1$ for dashed, $c=0.01$ for dotted, and $c=0.001$ for solid curves.

\section{1 $c<0$ GBAdS black holes: Not well-defined}

As is shown in Fig. 2, this $c<0$ case shows a typical behavior of the negative mass, negative temperature, and negative entropy except the $r_{h}>l$ case of BBH. Explicitly, we have $\mu<0$ for $r_{h}<l_{0}=(l / \sqrt{2})\left[-1+\left(1-4 c / l^{2}\right)^{1 / 2}\right]^{1 / 2}$, $T<0$ for $r_{h}<r_{c}=\sqrt{-2 c}$ and $S<0$ for $r_{h}<\sqrt{-6 c}$. These negative values persist to any negative coupling. This implies that the thermodynamics is not well-defined for the $c<0$ GBAdS. We call these the improper black hole because these can not pass under the thermodynamic test.

The $c=0$ case is the SAdS whose temperature is given by

$$
T_{H}^{S A d S}\left(r_{h}\right)=\frac{r_{h}}{\pi l^{2}}\left(1+\frac{l^{2}}{2 r_{h}^{2}}\right) .
$$

In this case, the entropy, heat capacity, and free energy are given by

$$
\begin{aligned}
S_{B H}^{S A d S}\left(r_{h}\right) & =\frac{\mathcal{A}_{3} r_{h}^{3}}{4 G_{5}} \\
C^{S A d S}\left(r_{h}\right) & =\frac{3 \mathcal{A}_{3} r_{h}^{3}}{4 G_{5}}\left[\frac{2 r_{h}^{2}+l^{2}}{2 r_{h}^{2}-l^{2}}\right] \\
F^{S A d S}\left(r_{h}\right) & =-\frac{\mathcal{A}_{3} r_{h}^{2}}{16 \pi G_{5}}\left[\frac{r_{h}^{2}}{l^{2}}-1\right]
\end{aligned}
$$

For the SAdS case, we have a typical form of heat capacity, showing the change from $-\infty$ to $\infty$ at the minimum temperature point of $r_{0}=l / \sqrt{2}$. Hence, this case has two phases of negative $(-)$ and positive $(+)$ heat capacities. 
Let us check whether in the limit of $c \rightarrow 0^{-}$, the temperature $T_{H}$ could reduce to that of the SAdS. At the first sight, the mathematical expression of Eq. (16) superficially seems to lead to Eq. (20). However, as depicted in Fig. 3-a, we cannot arrive at the SAdS case as $c \rightarrow 0^{-}$because the negative temperature always appears unless $c=0$. The same also works for mass

$\mu$ and entropy $S_{B H}$. This feature persists in all $c<0$ black holes. As a result, these confirm thermodynamically that the SAdS black hole could not be continuously reduced from the GBAdS.

\section{$3.2 \quad c>0$ GBAdS black holes}

For the $c>0$ case inspired by string theories, we have completely different black holes. Since the extremal black holes are defined to have zero temperature, let us check that there is an extremal solution at $r_{h}=r_{e}=0$ with $\mu=\frac{3}{2} c$. In this case, we extract out important points of the Davies' point (D) $[32,33,34]$ and minimum temperature point (0) from the condition of $d T / d r_{h}=0$ [35],

$$
r_{D / 0}^{2}=3\left[\left(\frac{l^{2}}{12}-c\right) \mp \sqrt{\left(c-l^{2} / 4\right)\left(c-l^{2} / 36\right)}\right] .
$$

These two points appear for the $0<c<l^{2} / 36$ case, while the critical (inflection) point appears at $r_{h}^{2}=l^{2} / 6$ for $c=l^{2} / 36$, and any point does not exist for $l^{2} / 36<c \leq l^{2} / 4$. Hence, we expect that the BIAdS-like black hole is for $0<c<l^{2} / 36$ [12], the critical GBAdS is for $c=l^{2} / 36$, and the NBTZ-like black hole is for $l^{2} / 36<c \leq l^{2} / 4$ [36]. The global features of the Hawking temperature depending on the parameter $c>0$ are shown in Fig. 4.

In the case of $0<c<l^{2} / 36$, the two of Davies' point and local minimum points appears. The graphs of the heat capacity depending on the parameter $c>0$ are shown in Fig. 5. We find that three phases of +-+ for $0<c<$ $l^{2} / 36$ with two blow-up points at Davies' and minimum points (See Fig.5a). Here we define four different black holes [15]: extremal black hole with zero heat capacity $\left(\mathrm{EBH}_{0}\right)$, small black hole with positive heat capacity $\left(\mathrm{SBH}_{+}\right)$; intermediate black hole with negative heat capacity $\left(\mathrm{IBH}_{-}\right)$; large black hole with positive heat capacity $\left(\mathrm{LBH}_{+}\right)$. In fact, these play the important roles in connection with the dynamical stability. Furthermore, the $\delta$-function phase appears for $c=l^{2} / 36$ (See Fig.5b) and a single positive phase appears for $l^{2} / 36<c \leq l^{2} / 4$ (See Fig.5c and Fig.5d).

We note that for $r_{h}>l$, we obtain the big black hole $\left(\mathrm{BBH}_{+}\right)$as the 


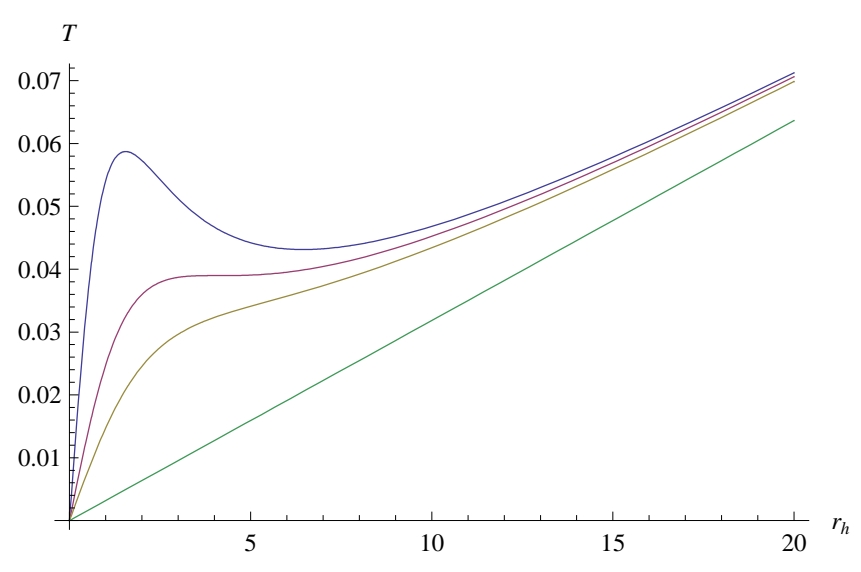

Figure 4: Temperature graphs $T$ vs $r_{h}$ with $l=10$ for $c>0$ from top to bottom: (a) $c=1$ ( $b Q=0.5$ BIAdS) (b) $c=25 / 9$ (critical case), (c) $c=5$, (d) $c=25$ (NBTZ). All temperatures are zero at the extremal point of $r_{e}=0$.

asymptotic forms of the GBAdS and SAdS

$$
T_{H}^{B B H}=\frac{r_{h}}{\pi l^{2}}, S_{B H}^{B B H}=\frac{\mathcal{A}_{3} r_{h}^{3}}{4 G_{5}}, C^{B B H}=3 S_{B H}^{B}, F^{B B H}=-\frac{\mathcal{A}_{3} r_{h}^{4}}{16 \pi G_{5} l^{2}} .
$$

These quantities are very useful for discussing the connection between thermodynamic stability and QN modes. For this $\mathrm{BBH}_{+}$, the global thermodynamic stability is guaranteed because of $C^{B B H}>0$ and $F^{B B H}<0$. However, for large black hole $\left(\mathrm{LBH}_{+}\right)$, the global thermodynamic stability is not guaranteed because for $r_{0}<r_{h}<r_{1}$ one has $C>0$ as shown in Fig. 5 and $F>0$ as shown in Fig. 6. Here $r_{1}\left(r_{0}\right)$ is the largest (next smaller) root determined from the condition of $F=0$.

\section{Thermodynamic stability and dynamical sta- bility}

In this section we discuss the connection between thermodynamic and dynamical stability [37]. This is known as the correlated stability conjecture $[38,39]$. First of all, the criterion of local thermodynamic stability is that $C>0(C<0)$ denote stable (unstable) black holes. On the other hand, the dynamical stability is determined by investigating the time-independent $s$-mode perturbation around the GBAdS background. These perturbations are given by

$$
h_{a}{ }^{b}=\operatorname{diag}\left[H_{t t}(r), H_{r r}(r), K(r), K(r), K(r)\right] .
$$



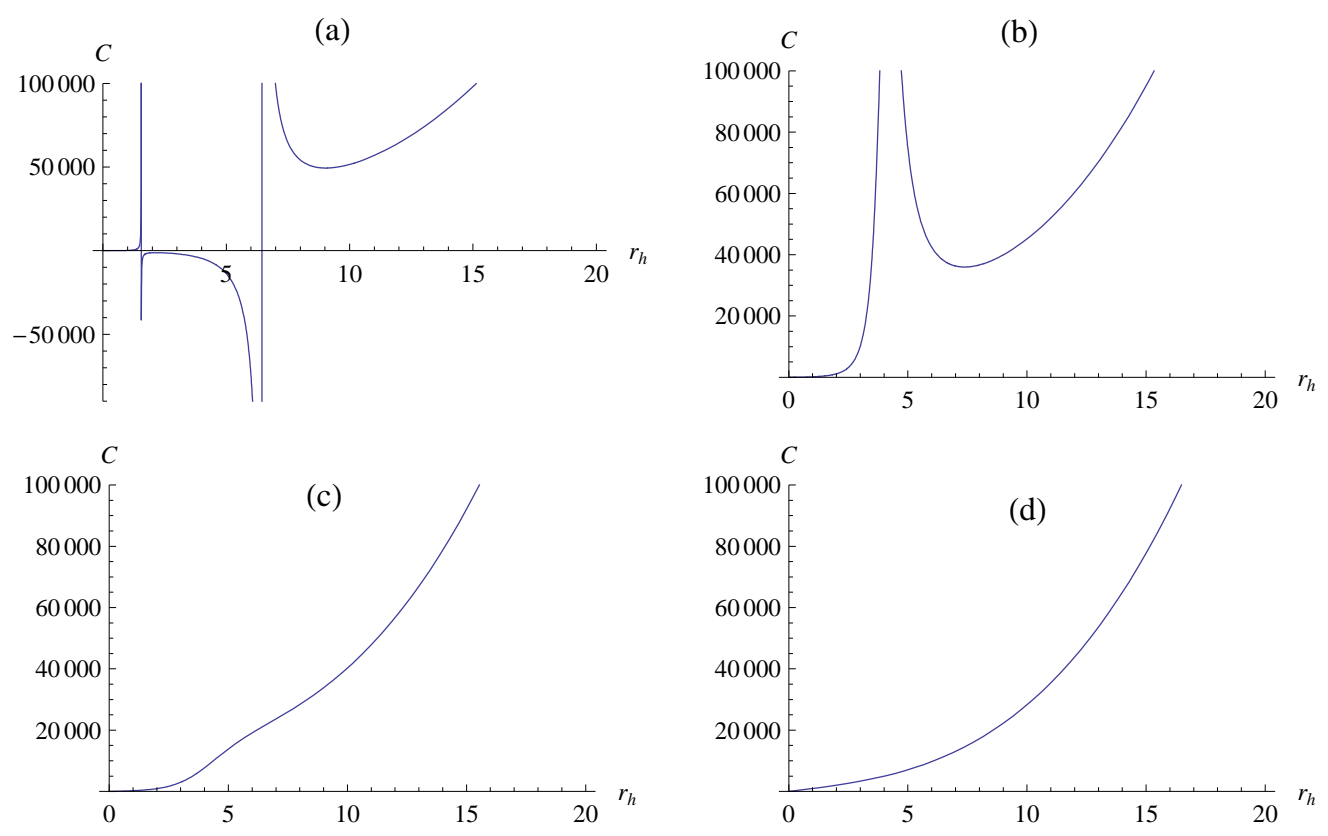

Figure 5: Heat capacity graphs $C$ vs $r_{h}$ with $l=10$ for $c>0$ : (a) $c=1$ $(b Q=0.5$ BIAdS) (b) $c=25 / 9$ (critical case), (c) $c=5$, (d) $c=25$ (NBTZ).

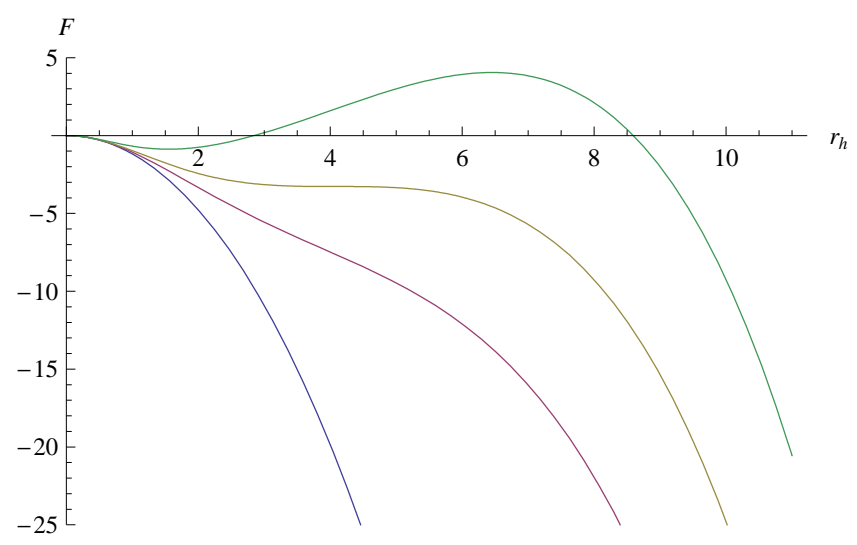

Figure 6: Free energy graphs $F$ vs $r_{h}$ with $l=10$ for $c>0$ from top to bottom: (a) $c=1$ ( $b Q=0.5$ BIAdS) (b) $c=25 / 9$ (critical case), (c) $c=5$, (d) $c=25$ (NBTZ).

In the Einstein gravity, there exist two independent modes, which are trace and traceless modes. The former is related to a conformal rescaling and it has a ghost-like kinetic term, while the latter gives negative modes. In order to compute the eigenvalues $\lambda$ of the Lichnerowicz operator $\Delta$, one should 
solve the tensor eigenvalue equation

$$
\Delta_{b d}^{a c} h_{c}^{d}=\lambda h_{b}^{a}
$$

with proper boundary conditions at infinity and horizon. Recently, Hirayama has studied the asymptotic behaviors of the $s$-mode perturbation at the horizon and the infinity in $r$, and solved this equation numerically [25]. Hence, we simply use this data to discuss the connection between dynamical and thermal stability. In this case, the criterion of dynamical stability means that $\lambda>0(\lambda<0)$ denote stable (unstable) black holes. In this section, we separate the whole region of $c$ into the following three cases because each case has different phase for the heat capacity.

\section{1 $c<0$ GBAdS black hole}

In this case, we may have a direct connection between thermodynamic and dynamic stability [25]. We have the $\mathrm{SBH}_{-}$for $r_{c}<r_{h}<r_{0}$, while the $\mathrm{LBH}_{+}$ appears for $r_{h}>r_{0}$. On the dynamical perturbation side, we may have the same result, which shows the unstable black hole with $\lambda<0$ for $r_{c}<r_{h}<r_{0}$ and the stable black hole with $\lambda>0$ for $r_{h}>r_{0}$. However, the connections of $C>0 \leftrightarrow \lambda>0$ and $C<0 \leftrightarrow \lambda<0$ are meaningless because this black hole is not well-defined thermodynamically. This clearly modifies the result of Ref. [25], which stated that the correlated stability conjecture holds for the $c<0$ GBAdS black hole.

\section{2 $0<c<l^{2} / 36$ GBAdS black holes}

The $0<c<l^{2} / 36$ case seems to not provide a direct connection between thermodynamic and dynamical stability [25] because this case has four different phases of the heat capacity [15]. These are $\mathrm{EBH}_{0}, \mathrm{SBH}_{+}, \mathrm{IBH}_{-}$, and $\mathrm{LBH}_{+}$. Up to now, we regard the $\mathrm{EBH}_{0}$, extremal black hole with zero heat capacity as the stable remnant of black hole. According to the perturbation calculation, the corresponding eigenvalue is a positive constant, which confirms that the $\mathrm{EBH}_{0}$ is thermodynamically stable even it has zero heat capacity. For the $\mathrm{LBH}_{+}$, there exists a direct connection of $C>0 \leftrightarrow \lambda>0$. However, there is no such relation for the $\mathrm{SBH}_{+}$and $\mathrm{IBH}_{-}$cases. Thermodynamically, the $\mathrm{SBH}_{+}$is quite different from $\mathrm{SBH}_{-}$. The former appears as the near-extremal black hole in the RNAdS, while the latter is the small unstable black hole, mediating the Hawking-Page transition (HP2) in the SAdS. On 
the other hand, the $\mathrm{IBH}_{-}$appears the intermediate unstable black hole in the RNAdS.

Then, one may conjecture that two black holes of the $\mathrm{SBH}_{+}$and $\mathrm{IBH}_{-}$are closely related to the unexplored transition at the Davies' point $\left(r_{h}=r_{D}\right)$. Although the $\mathrm{SBH}_{+}$is thermodynamically stable, while the $\mathrm{IBH}_{-}$is unstable as the index indicates, there is no single value for $\lambda$ for these cases on the perturbation side. That is, one has multiple values of $\left\{\lambda_{i}\right\}$ for a given horizon radius $r_{h}$ around the Davies' point. Hence we do not confirm the connection between thermodynamic and dynamical stability for the $\mathrm{SBH}_{+}$and $\mathrm{IBH}_{-}$ cases. This means that the Davies' point is quite different from the minimum temperature point $\left(r_{h}=r_{0}\right)$ even they give rise to the same blow-up of the heat capacity in the GBAdS [15] and RNAdS [12]. Hence, we do not make any decisive connection between thermodynamic and dynamical stability for the $0<c<l^{2} / 36$ GBAdS. Therefore, the correlated stability conjecture does not work for this black hole, as it does not work for the RNAdS.

\section{3 $l^{2} / 36<c \leq l^{2} / 4$ GBAdS black holes}

We have a single phase only for the $l^{2} / 36<c \leq l^{2} / 4$ GBAdS. This case is simple and similar to that of the NBTZ without $\mathrm{SBH}_{-}$. Although the $\mathrm{SBH}_{-}$ plays a role of the mediator in the HP2, it is absent here. The connection is clearly defined: thermodynamically stable black hole $(C>0) \longleftrightarrow$ stable black hole ( $\lambda=$ positive constant) [25]. The correlated stability conjecture does work for this black hole well.

\section{Thermodynamic stability and QN modes}

First, let us discuss the thermodynamic fluctuations of black holes. There exist already several works, which show that for a large black hole in AdS spacetimes, the Bekenstein-Hawking entropy receives logarithmic corrections due to thermodynamic fluctuations [40, 41, 42, 43, 44, 45]. The suggested formula takes the form

$$
S=S_{B H}-\frac{1}{2} \ln [C]+\cdots,
$$

where $C$ is the heat capacity of Eq. (17) and $S_{B H}$ denotes the uncorrected Bekenstein-Hawking entropy in Eq. (18). Here, an important point is that in order for Eq. (28) to make sense, $C$ should be positive. Hence, we can not make any correction to the entropy for the $\mathrm{EBH}_{0}$ and $\mathrm{IBH}_{-}$, while we 
could make correction to the $\mathrm{SBH}_{+}$and $\mathrm{LBH}_{+}$. As a limiting case, the 5D Schwarzschild black hole, which is asymptotically flat, has a negative heat capacity of $C^{S c h}=-3 S_{B H}^{S c h}[46]$. This means that the Schwarzschild black hole is never in thermal equilibrium and it is always unstable against thermal fluctuations. Thus, we do not make any correction to the entropy of the Schwarzschild black hole. On the other hand, we have $C>0$ for the BTZ black hole, which shows that the entropy correction is always possible to occur. This means that the thermodynamic fluctuations on the black hole with the positive heat capacity leads to the logarithmic correction to the Bekenstein-Hawking entropy.

On the other hand, it is well known that if one perturbs a black hole, the surrounding geometry will ring (undergo damped oscillations) [16]. These damped oscillations known as "quasinormal(QN) modes" are entirely fixed by the thermodynamic quantities of black hole and are independent of the initial perturbations. In general, their $\mathrm{QN}$ frequencies are complex and discrete,

$$
\omega=\omega_{R}-i \omega_{I}
$$

which describe the decay of external perturbations outside the event horizon. However, as far as we know, their analytic expressions are known for two limited cases: NBTZ in three dimensions [17] and topological massless black hole (TMBH) in higher dimensions [18]. For the big black holes in AdS spaces, the QN frequencies are known to take analytic form approximately [19, 20]. However, for small and intermediate black holes, there exists no analytic form. This suggests a close connection between the thermodynamic stability and QN modes.

It seems appropriate to mention the connection between global thermodynamic stability and QN modes for BBHs in AdS spacetimes. If $C>0$, the global thermodynamic stability (GTS) is guaranteed by the condition of a negative free energy $F<0$. Actually, the $\mathrm{BBH}_{+}$with $r_{h}>l$ satisfies the GTS. As shown in Eq. (25), this case always has negative free energy. Hence, we conjecture the important connection between GTS and QN modes:

If a black hole is big and thus globally stable, its QN modes may take analytic expressions.

The typical examples are 3D NBTZ and 5D TMBH for completely analytic expressions and the big SAdS for approximately analytic expressions. The first two expressions for scalar perturbations are given by $[17,18,20,21]$

$$
\begin{aligned}
\omega_{s}^{N B T Z} & =4 \pi T_{H}^{N B T Z}[ \pm \ell-i(n+1)] \\
\omega_{s}^{T M B H} & =2 \pi T_{H}^{T M B H}\left[ \pm \xi_{s}^{T M B H}-2 i\left(n+\frac{1}{2}\right)\right]
\end{aligned}
$$


where $T_{H}^{N B T Z}=\frac{r_{h}}{2 \pi l^{2}}$, $\ell$ with $\nabla^{2} Y=-\ell^{2} Y$ on $S^{1}$ and $T_{H}^{N B T Z}=\frac{1}{2 \pi l}$, $\xi_{s}^{T M B H}=\sqrt{k_{s}^{2}-1 / 4}$ with $\nabla^{2} Y=-k_{s}^{2} Y$ on $S^{3}$. For the NBTZ, we have a massless scalar, while we consider the massive scalar with $m^{2} l^{2}=-4$ for the TMBH. The $\mathrm{QN}$ frequencies for $\mathrm{BBH}_{+}$takes the following form for a massless scalar [22]:

$$
\omega_{s}^{B B H} \simeq 8 \pi T_{H}^{B B H}[ \pm n-i n]
$$

where $T_{H}^{B B H}=\frac{r_{h}}{\pi l^{2}}$.

Parallel to the previous section, we also separate the whole region of $c$ into the following three cases.

\section{$5.1 \quad c<0$ GBAdS black holes}

In this case, we may have a connection between thermodynamic stability and QN modes for the $\mathrm{BBH}_{+}$. We assume that QN frequencies take approximately analytic form. However, as emphasized before, this black hole is not well-defined thermodynamically. Hence, it is meaningless to consider this connection for the $c<0$ GBAdS black hole.

\section{$\mathbf{5 . 2} 0<c \leq l^{2} / 36$ GBAdS black holes}

For the $\mathrm{SBH}_{+}$and $\mathrm{IBH}_{-}$cases, we can not expect to have analytic form of QN frequencies because their connection between thermodynamic and dynamical stabilities is not yet established. However, for the $\mathrm{BBH}_{+}$, we may have approximately analytic form of QN frequencies like Eq. (32). Finally, we expect that the QN frequencies are changed drastically around the Davies' point for this case because the same thing happens for the RNAdS [34, 23].

\section{3 $l^{2} / 36<c \leq l^{2} / 4$ GBAdS black holes}

We expect that its QN modes take analytic form because this case has a single phase of the positive heat capacity. Specifically, for the upper bound case of $c=l^{2} / 4$, we strongly expect to have the presence of analytic expressions like Eq. (30) because this case corresponds to the NBTZ [17]. 
Table 1: The role of the Gauss-Bonnet coupling constant $c$ for various black holes.

\begin{tabular}{|c|c|c|c|}
\hline black holes & $c<0$ & $c>0$ & reference \\
\hline GBAdS & negative mass & pseudo-charge & SAdS/ BIAdS \\
TGBAdS & negative mass & $\cdot$ & TAdS/ $\cdot$ \\
GBRNAdS & $\cdot$ & charge & $\cdot /$ RNAdS \\
\hline
\end{tabular}

\section{Summary and discussions}

We have considered the GBAdS black hole in five dimensions to study its thermodynamics thoroughly. First of all, we have pointed out that this black hole does satisfy the first-law of thermodynamics, but its entropy does not satisfy the area-law. This is mainly because we have used the first-law to derive the entropy.

We could not find a thermodynamically well-defined black hole for the $c<0$ case where $c$ may play the role of negative mass. Although this seems to be the SAdS-type black hole, all of its mass, temperature, and entropy are negative unless $c=0$. Moreover, the role of negative mass " $c$ " becomes clear when introducing the $k=-1$ topological GBAdS (TGBAdS) in Appendix A.

On the other hand, for the $c>0$ case, the $b Q=0.5$ BIAdS will be used to study the $0<c<l^{2} / 36$ GBAdS because their thermodynamic properties are the nearly same. This corresponds to the black hole inspired by the string theories. In this case, $c$ plays the role of a pseudo-charge. The role of charge " $c$ " becomes clear when considering the GBRNAdS black hole in Appendix B. We clarify the role of " $c$ " in Table 1.

Furthermore, we could not obtain the SAdS in the limits of $c \rightarrow 0^{\mp}$. This contradicts to the view that the GBAdS is the deformed SAdS. The origin of this problem is the order of taking the $c \rightarrow 0$ limit in calculation of thermodynamic quantities. If this limit is taken before the calculation of thermodynamic quantities, then we find thermodynamic quantities of the SAdS very well. Actually, this corresponds to turning off the GB term $L_{G B}$ in the action. On the other hand, if this limit is taken after the calculation of thermodynamic quantities, then we could not find the thermodynamic quantities of the SAdS. This implies that the order of taking the $c \rightarrow 0$ limit is very important to recover the SAdS from the GBAdS.

In addition, we have clarified the connection between thermodynamic and dynamical stability, known as the correlated stability conjecture. We have found that this conjecture is valid for the $l^{2} / 36<c \leq l^{2} / 4$ GBAdS only because this case has a single positive phase of the heat capacity. On the 

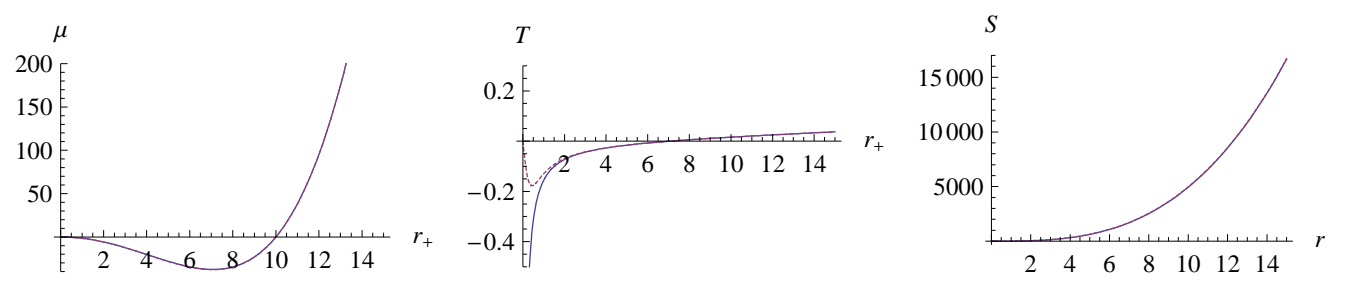

Figure 7: Thermodynamic quantities of mass $\mu$, temperature $T$, and entropy $S$ for $k=-1$ TGBAdS black hole. The dotted curves represent the $k=-1$ TAdS black hole with $c=0$, while the solid curves denote $k=-1$ TGBAdS with $c=-0.10$.

other hand, for the $0<c \leq l^{2} / 36$ GBAdS, this conjecture does not work because this has four different phases of the heat capacity, showing that it is not easy to make a direct connection between thermodynamic and dynamical stability.

Finally, we have newly proposed the important connection between the global thermodynamic stability (GTS: $C>0, F<0$ ) and the analytic expressions of QN frequencies: If the black hole satisfies the GTS, its QN modes may have the analytic form. The conjecture works for the black hole in asymptotically AdS spacetimes.

\section{Appendix A: Thermodynamic quantities of $k=$ -1 TGBAdS black holes}

In this case with $c<0$, we have the relevant thermodynamic quantities

$$
\begin{gathered}
\mu\left(r_{h}\right)=\frac{3}{2}\left(\frac{r_{h}^{4}}{l^{2}}-r_{h}^{2}+c\right), \\
T\left(r_{h}\right)=\frac{r_{h}^{3}}{\pi l^{2}\left(r_{h}^{2}-2 c\right)}\left(1-\frac{l^{2}}{2 r_{h}^{2}}\right), \\
S\left(r_{h}\right)=\frac{\mathcal{A}_{3} r_{h}^{3}}{4 G_{5}}\left(1-\frac{6 c}{r_{h}^{2}}\right),
\end{gathered}
$$

The important observation is that there is no blow-up point of $T$ in Eq. (A2), and the entropy is always positive as is shown in Eq. (A3). These improve the troubles related to the blow-up temperature and the negative entropy in the $c<0$ GBAdS black hole. These are depicted in Fig. 7 . 

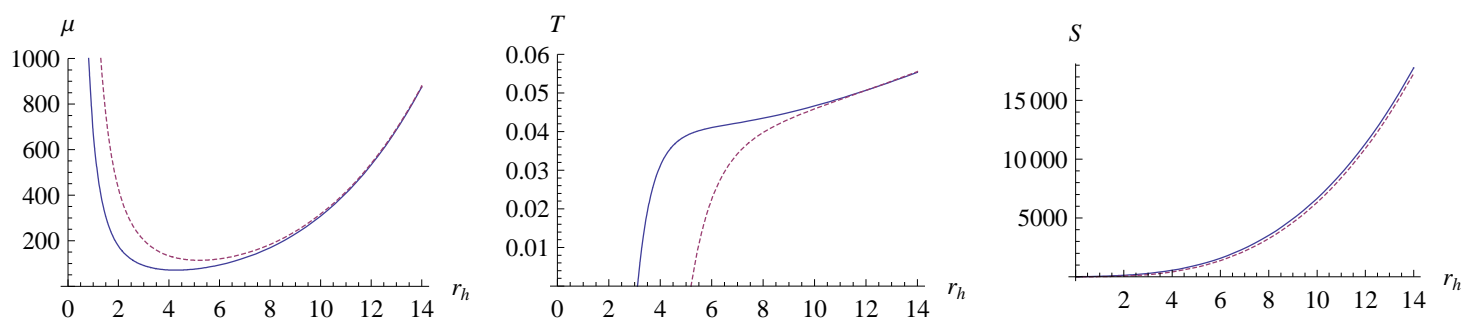

Figure 8: Thermodynamic quantities of mass $\mu$, temperature $T$, and entropy $S$ for GBRNAdS black hole. The dotted curves represent the RNAdS black hole with $c=0$, while the solid curves denote GBRNAdS with $c=0.1$. Two shows the nearly same behavior.

\section{Appendix B: Thermodynamic quantities of GBR- NAdS black holes}

In this case with $c>0$, the metric function takes the form

$$
f(r)=1+\frac{r^{2}}{2 c}\left(1-\sqrt{1+\frac{2 c}{3}\left(\frac{4 \mu}{r^{4}}-\frac{6}{l^{2}}\right)-\frac{9 q^{2}}{2 r^{6}}}\right),
$$

which is obtained by adding $-\frac{1}{16 \pi G_{5}} F^{2}$ to the action (1). Then, the relevant thermodynamic quantities of mass $\mu$, temperature $T$, and the entropy $S$ are given by [28]

$$
\begin{gathered}
\mu\left(r_{h}, c, q\right)=\frac{3}{2}\left(\frac{r_{h}^{4}}{l^{2}}+r_{h}^{2}+\frac{9 q^{2}}{8 c} \frac{1}{r_{h}^{2}}\right), \\
T\left(r_{h}, c, q\right)=\frac{r_{h}^{3}}{\pi l^{2}\left(r_{h}^{2}+2 c\right)}\left(1+\frac{l^{2}}{2 r_{h}^{2}}-\frac{9 q^{2}}{16 c} \frac{l^{2}}{r_{h}^{6}}\right), \\
S\left(r_{h}, c, q\right)=\frac{\pi^{2}}{2} r_{h}^{3}\left(1+\frac{6 c}{r_{h}^{2}}\right) .
\end{gathered}
$$

From Fig. 8, we observe the role of $c$ as the square of charge $q^{2}$ because it appears as a combination of $q^{2} / c$ in Eqs. (B2) and (B3).

\section{Acknowledgments}

Y. S. Myung was supported by the Korea Research Foundation (KRF-2006311-C00249) funded by the Korea Government (MOEHRD). Y.-W. Kim was 
supported by the Korea Research Foundation Grant funded by Korea Government (MOEHRD): KRF-2007-359-C00007. Y.-J. Park was supported by the Korea Science and Engineering Foundation (KOSEF) grant funded by the Korea government (MOST) (R01-2007-000-20062-0).

\section{References}

[1] D. G. Boulware and S. Deser, "String Generated Gravity Models," Phys. Rev. Lett. 55, 2656 (1985).

[2] R. C. Myers and J. Z. Simon, "Black Hole Thermodynamics in Lovelock Gravity," Phys. Rev. D 38, 2434 (1988).

[3] D. J. Gross and E. Witten, "Superstring Modifications Of Einstein's Equations," Nucl. Phys. B 277, 1 (1986);

R. R. Metsaev and A. A. Tseytlin, "Two loop beta function for the generalized bosonic sigma model," Phys. Lett. B 191, 354 (1987);

C. G. Callan, R. C. Myers, and M. J. Perry, "Black Holes in String Theory," Nucl. Phys. B 311, 673 (1989).

[4] R. C. Myers, "Higher derivative gravity, surface terms and string theory," Phys. Rev. D 36, 392 (1987).

[5] J. T. Wheeler, "Symmetric Solutions To The Gauss-Bonnet Extended Einstein Equations," Nucl. Phys. B 268, 737 (1986).

[6] Y. M. Cho and I. P. Neupane, "Anti-de Sitter black holes, thermal phase transition and holography in higher curvature gravity," Phys. Rev. D 66, 024044 (2002) [arXiv:hep-th/0202140].

[7] T. Torii and H. Maeda, "Spacetime structure of static solutions in Gauss-Bonnet gravity: Neutral case," Phys. Rev. D 71, 124002 (2005) [arXiv:hep-th/0504127].

[8] S. W. Hawking and D. N. Page, "Thermodynamics of black holes in anti-de Sitter space," Commun. Math. Phys. 87, 577 (1983).

[9] E. Witten, "Anti-de Sitter space, thermal phase transition, and confinement in gauge theories," Adv. Theor. Math. Phys. 2, 505 (1998) [arXiv:hep-th/9803131]. 
[10] A. Chamblin, R. Emparan, C. V. Johnson, and R. C. Myers, "Charged AdS black holes and catastrophic holography," Phys. Rev. D 60, 064018 (1999) [arXiv:hep-th/9902170].

[11] T. K. Dey, S. Mukherji, S. Mukhopadhyay, and S. Sarkar, "Phase transitions in higher derivative gravity and gauge theory: R-charged black holes," JHEP 0709, 026 (2007) [arXiv:0706.3996 [hep-th]].

[12] Y. S. Myung, "Phase transition between non-extremal and extremal Reissner-Nordstrom black holes", Mod. Phys. Lett. A 23, 667 (2008) [arXiv:0710.2568 [gr-qc]].

[13] T. K. Dey, S. Mukherji, S. Mukhopadhyay, and S. Sarkar, "Phase Transitions in Higher Derivative Gravity," JHEP 0704, 014 (2007) [arXiv:hepth/0609038].

[14] Y. S. Myung, Y.-W. Kim, and Y.-J. Park, "Thermodynamics of Einstein-Born-Infeld black holes in three dimensions," Phys. Rev. D (in press) arXiv:0804.0301 [gr-qc].

[15] Y. S. Myung, Y.-W. Kim and Y.-J. Park, "Thermodynamics and phase transitions in the Born-Infeld-anti-de Sitter black holes," arXiv:0805.0187 [gr-qc].

[16] K. D. Kokkotas and B. G. Schmidt, "Quasi-normal modes of stars and black holes," Living Rev. Rel. 2, 2 (1999) [arXiv:gr-qc/9909058].

[17] D. Birmingham, I. Sachs, and S. N. Solodukhin, "Conformal field theory interpretation of black hole quasi-normal modes," Phys. Rev. Lett. 88, 151301 (2002) [arXiv:hep-th/0112055].

[18] D. Birmingham and S. Mokhtari, "Exact gravitational quasinormal frequencies of topological black holes," Phys. Rev. D 74, 084026 (2006) [arXiv:hep-th/0609028].

[19] G. T. Horowitz and V. E. Hubeny, "Quasinormal modes of AdS black holes and the approach to thermal equilibrium," Phys. Rev. D 62, 024027 (2000) [arXiv:hep-th/9909056].

[20] G. Siopsis, "Analytic calculation of quasi-normal modes," arXiv:0804.2713 [hep-th].

[21] Y. S. Myung and H. W. Lee, "Unitarity issue in BTZ black holes," Mod. Phys. Lett. A 21, 1737 (2006) [arXiv:hep-th/0506031]. 
[22] S. Musiri and G. Siopsis, "Asymptotic form of quasi-normal modes of large AdS black holes," Phys. Lett. B 576, 309 (2003) [arXiv:hepth/0308196].

[23] E. Berti and V. Cardoso, "Quasinormal modes and thermodynamic phase transitions," Phys. Rev. D 77, 087501 (2008) [arXiv:0802.1889 [hep-th]].

[24] J. Grain and A. Barrau, Eur. Phys. J. C 53, 641 (2008) [arXiv:hepth/0701265].

[25] T. Hirayama, "Negative modes of Schwarzschild black hole in EinsteinGauss-Bonnet Theory," arXiv:0804.3694 [gr-qc].

[26] R. Myers, "Superstring gravity and black holes," Nucl. Phys. B 289, 701 (1987).

[27] S. Nojiri and S. D. Odintsov, "Anti-de Sitter black hole thermodynamics in higher derivative gravity and new confining-deconfining phases in dual CFT," Phys. Lett. B 521, 87 (2001) [Erratum-ibid. B 542, 301 (2002)] [arXiv:hep-th/0109122].

[28] R. G. Cai, "Gauss-Bonnet black holes in AdS spaces," Phys. Rev. D 65, 084014 (2002) [arXiv:hep-th/0109133].

[29] M. Cvetic, S. Nojiri, and S. D. Odintsov, "Black hole thermodynamics and negative entropy in deSitter and anti-deSitter Einstein-GaussBonnet gravity," Nucl. Phys. B 628, 295 (2002) [arXiv:hep-th/0112045];

[30] I. P. Neupane, "Black hole entropy in string-generated gravity models," Phys. Rev. D 67, 061501 (2003) [arXiv:hep-th/0212092].

I. P. Neupane, "Thermodynamic and gravitational instability on hyperbolic spaces," Phys. Rev. D 69, 084011 (2004) [arXiv:hep-th/0302132].

[31] T. Clunan, S. F. Ross, and D. J. Smith, "On Gauss-Bonnet black hole entropy," Class. Quant. Grav. 21, 3447 (2004) [arXiv:gr-qc/0402044].

[32] P. C. W. Davies, "The Thermodynamic Theory of Black Holes," Proc. R. Soc. Lond. A 353, 499 (1977).

[33] D. Pavon, "Phase transition in Reissner-Nordström black holes," Phys. Rev. D 43, 2495 (1991).

[34] J. Jing and Q. Pan, "Quasinormal modes and second order thermodynamic phase transition for Reissner-Nordström black hole," Phys. Lett. B 660, 13 (2008) [arXiv:0802.0043 [gr-qc]]. 
[35] Y. S. Myung, Y.-W. Kim, and Y.-J. Park, "Ruppeiner geometry and 2D dilaton gravity in the thermodynamics of black holes," Phys. Lett. B 663, 342 (2008) [arXiv:0802.2152 [hep-th]].

[36] Y. S. Myung, "Phase transition between the BTZ black hole and AdS space," Phys. Lett. B 638, 515 (2006) [arXiv:gr-qc/0603051].

[37] T. Harmark, V. Niarchos, and N. A. Obers, "Instabilities of black strings and branes," Class. Quant. Grav. 24, R1 (2007) [arXiv:hep-th/0701022].

[38] S. S. Gubser and I. Mitra, "Instability of charged black holes in anti-de Sitter space," arXiv:hep-th/0009126;

S. S. Gubser and I. Mitra, "The evolution of unstable black holes in anti-de Sitter space," JHEP 0108, 018 (2001) [arXiv:hep-th/0011127].

[39] H. S. Reall, "Classical and thermodynamic stability of black branes," Phys. Rev. D 64, 044005 (2001) [arXiv:hep-th/0104071].

[40] R. K. Kaul and P. Majumdar, "Quantum black hole entropy," Phys. Lett. B 439, 267 (1998) [arXiv:gr-qc/9801080].

[41] R. K. Kaul and P. Majumdar, "Logarithmic correction to the Bekenstein-Hawking entropy," Phys. Rev. Lett. 84, 5255 (2000) [arXiv:gr-qc/0002040].

[42] S. Carlip, "Logarithmic corrections to black hole entropy from the Cardy formula," Class. Quant. Grav. 17, 4175 (2000) [arXiv:gr-qc/0005017].

[43] T. R. Govindarajan, R. K. Kaul, and V. Suneeta, "Logarithmic correction to the Bekenstein-Hawking entropy of the BTZ black Class. Quant. Grav. 18, 2877 (2001) [arXiv:gr-qc/0104010].

[44] D. Birmingham and S. Sen, "An exact black hole entropy bound," Phys. Rev. D 63, 047501 (2001) [arXiv:hep-th/0008051].

[45] R. Banerjee and B. R. Majhi, "Quantum Tunneling and Back Reaction," Phys. Lett. B 662, 62 (2008) [arXiv:0801.0200 [hep-th]];

R. Banerjee and B. R. Majhi, "Quantum Tunneling Beyond Semiclassical Approximation," arXiv:0805.2220 [hep-th].

[46] S. Das, P. Majumdar, and R. K. Bhaduri, "General logarithmic corrections to black hole entropy," Class. Quant. Grav. 19, 2355 (2002) [arXiv:hep-th/0111001]. 